\title{
URINARY TRACT INFECTION: PRESCRIBING PATTERN OF ANTIBIOTICS AT A TERTIARY CARE HOSPITAL
}

\author{
PANAYAPPAN L*, ATHIRA SURESH BABU, DEENA DAVIS, NEETHU JOSEPH, NEETHU JOSHY, KRISHNAKUMAR K
}

Department of Pharmacy Practice, St. James College of Pharmaceutical Sciences, Thrissur, Kerala, India. Email: stjamespharmacyproject@gmail.com

Received: 25 January 2017, Revised and Accepted: 22 February 2017

\section{ABSTRACT}

Objectives: The purpose of drug utilization review is to ensure drugs are used appropriately, safely, and effectively to improve patient health status. The use of drugs in appropriate, safe, and effective manner would decrease the treatment cost for the patients. Urinary tract infection (UTI) is most common and can lead to secondary infections. Hence, continuous analysis of prescribing pattern in UTI is a vital one. With this information, the study was designed to determine various risk factors and prescribing pattern of rational use of antibiotics in UTI patients at a tertiary care hospital.

Methods: A prospective observational study was carried out in 100 patients in a tertiary care teaching hospital, by collecting patient data from the medical records of patients from General Medicine Department.

Results: Analysis of prescribed drugs revealed that use of antibiotics such as ceftriaxone (35\%) and amikacin (22\%) were significantly higher than other drugs prescribed such as ciprofloxacin (16\%), nitrofurantoin (15\%), ofloxacin (5\%), cefixime (3\%), moxifloxacin (2\%), and clarithromycin (2\%). Culture sensitivity test was done only in $30 \%$ of the total cases (100) for which empirical treatment had to be applied rather than specific antibiotic treatment. The most common isolated organisms were Escherichia coli (60\%), Proteus (20\%), Klebsiella (13.33\%), and Pseudomonas (6.66\%).

Conclusion: Cephalosporins, aminoglycosides, and quinolones were the most commonly prescribed antibiotics in this study. Present findings together with previous ones are suggestive of the need for periodic monitoring of antibiotic sensitivity pattern of the bacterial isolates to provide effective treatment.

Keywords: Antibiotics, Prescription, Bacterial infection.

(C) 2017 The Authors. Published by Innovare Academic Sciences Pvt Ltd. This is an open access article under the CC BY license (http://creativecommons. org/licenses/by/4. 0/) DOI: http://dx.doi.org/10.22159/ajpcr.2017.v10i5.17287

\section{INTRODUCTION}

The purpose of drug utilization review is to ensure drugs are used appropriately, safely, and effectively to improve patient health status. The use of drugs in appropriate, safe, and effective manner would decrease the treatment cost for the patients. Drug utilization review is an authorized and structured ongoing review of practitioner prescribing, pharmacist dispensing, and patient use of medications [1]. Drug utilization program always plays an important role in helping the health-care team to improve the prescribing, administration, and use of medications. Pharmacists can then, in collaboration with prescribers and other members of the health-care team, initiate action to improve drug therapy for patients [2]. A urinary tract infection (UTI) is a condition in which one or more parts of the urinary system become infected. Each type of UTI may result in more specific signs and symptoms, depending on which part of the urinary tract is infected. UTIs are the most common of all bacterial infections and can occur at any time in the life of an individual [3]. The most common UTIs occur mainly in women and affect the bladder and urethra. The cystitis is the infection of the bladder and urethritis is the infection of the urethra. Furthermore, because the female urethra is close to the vagina, sexually transmitted infections, such as herpes, gonorrhea, chlamydia, and mycoplasma, can cause urethritis [4]. The various risk factors associated with UTIs are menopause, urinary tract abnormalities, birth control, catheter use, and a urinary procedure. There is no evidence that good hygiene makes a real difference in preventing UTIs, but it always makes a wise practice. Culture sensitivity tests were also important in case of UTI to go for a specific antibiotic treatment rather than the empirical treatment for the patients. The drug-prescribing patterns and common pathogens of the medicine outpatient department were identified by this study.

\section{METHODS}

\section{Ethics approval}

Ethical approval for the study was obtained from Institutional Human Ethics Committee (Ref: IHEC/SJCP/A.41/2015-2016), and the study was conducted at St. James Hospital, Chalakudy, Kerala. Informed written consent was obtained from all participants.

A prospective observational study was carried out in 100 patients in a tertiary care teaching hospital, by collecting patient data from the medical records of patients from General Medicine Department. Patients got admitted in General Medicine Department having UTI were selected. Both male and female patients in the age group of 18-80 years were included in the study. Patients with UTI not prescribed with antibiotics were excluded from the study.

\section{RESULTS}

Study on prescribing pattern and rational use of antibiotics in UTI patients in a tertiary care hospital was conducted. The study indicates the female patients (54\%) were more prone to UTI when compared to male patients (46\%) (Fig. 1).

The predominant risk factors to UTI were found to be diseases such as diabetes mellitus, benign prostatic hyperplasia, hypertension, dyslipidemia, and recurrent UTI, habit of smoking and alcoholism, and the use of oral contraceptive pill/intrauterine devices (Fig. 2).

Analysis of prescribed drugs revealed that use of antibiotics such as ceftriaxone (35.48\%) and amikacin (22.58\%) were significantly higher than other drugs prescribed such as ciprofloxacin $(16.12 \%)$, 


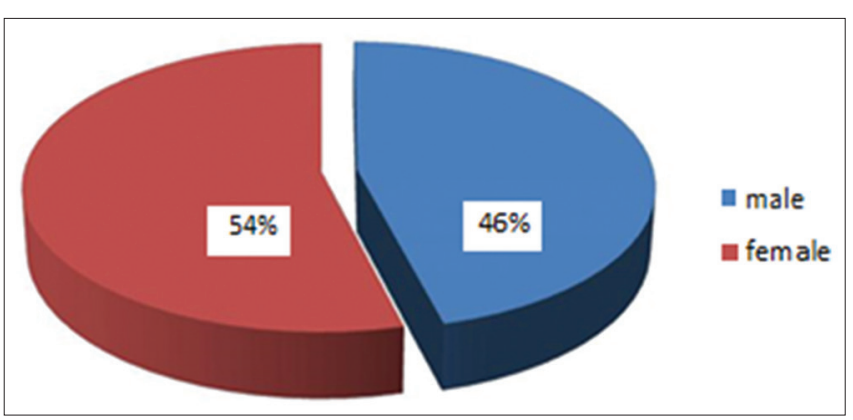

Fig. 1: Percentage of cases with gender distribution

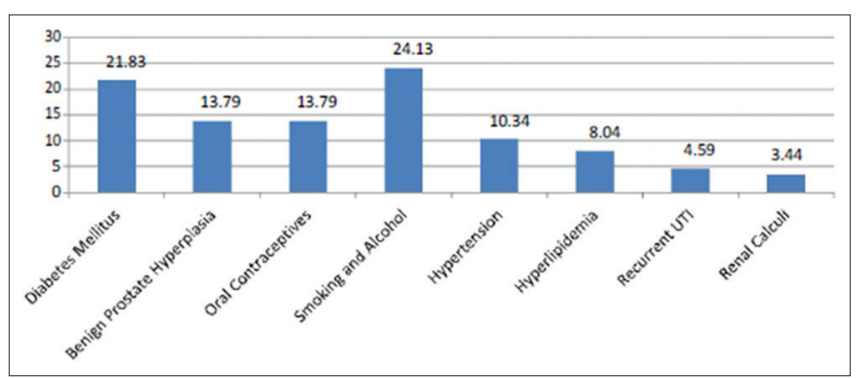

Fig. 2: Percentage distribution of patients according to adjuvant drugs prescribed along with antibiotics for urinary tract infection

nitrofurantoin (14.51\%), ofloxacin (4.83\%), cefixime (3.22\%), moxifloxacin (1.61\%), and clarithromycin (1.61\%) (Fig. 3).

Culture sensitivity test was done only in $30 \%$ of the total cases for which empirical treatment had to be applied rather than specific antibiotic treatment and the most common isolated organisms were Escherichia coli (60\%), Proteus (20\%), Klebsiella (13.33\%), and Pseudomonas (6.66\%) (Fig. 4).

\section{DISCUSSION}

In a study about National Patterns in the Treatment of UTI in Women by Ambulatory Care Physicians concludes that there are increasing in the use of fluoroquinolones and nitrofurantoin even though they are not highly recommended and not the most cost effective [5]. One more study about the management of uncomplicated UTI has concluded that trimethoprim-sulfamethoxazole and trimethoprim are still considered first-line therapy for uncomplicated UTI in areas where resistance in the community is $<10-20 \%$ [6]. Our study reveals the use of antibiotics as, out of 124 antibiotics used in 100 patients, ceftriaxone in 44 cases (35.48\%), amikacin in 28 cases $(22.58 \%)$ than ciprofloxacin in 20 cases (16.12\%), nitrofurantoin in 18 cases $(14.51 \%)$ followed by ofloxacin in 6 cases $(4.83 \%)$ and rest were prescribed as 4 cases $(3.22 \%)$ of cefixime and 2 cases $(1.61 \%)$ each of moxifloxacin and clarithromycin. In another study, it reveals that symptoms alone have low accuracy when assessed against the reference standard for diagnosing UTI. Empiric treatment of UTI based on symptoms may expose large number of patients to unnecessary antibiotics [7]. A study concluded about antibiotic resistance patterns of outpatient pediatric UTI and has concluded that E. coli remains the most common pediatric uropathogen [8]. Prospective observational study on prescribing pattern of antimicrobial agents in a tertiary care teaching hospital was conducted, and this study indicated that there is a need for motivating the physicians to prescribe antimicrobial agents by generic names with supportive bacteriological evidence [9]. A prospective observational study was carried out on the excessive and inappropriate use of antibiotics in a Tertiary Care Teaching Hospital for 6 months in General Medicine and Pediatrics Department. This study results indicated that cephalosporin $(72.72 \%)$ were mostly prescribed to inpatients, and penicillin's (57.29\%) usage was found

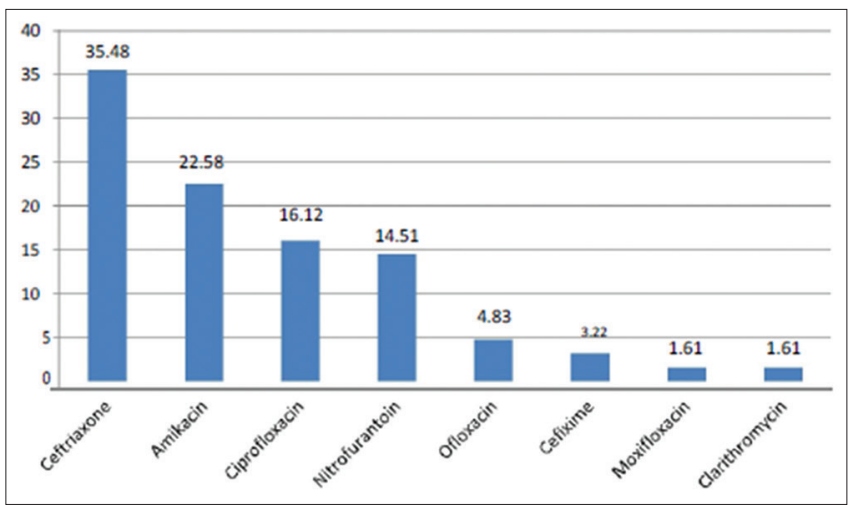

Fig. 3: Percentage distribution of patients according to antibiotic prescribed for urinary tract infection

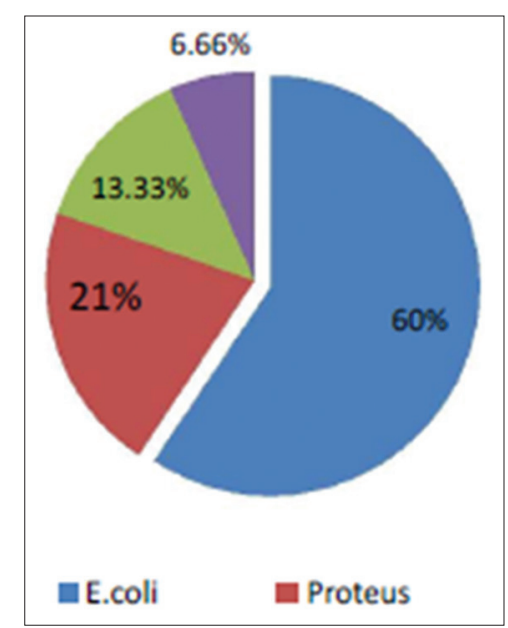

Fig. 4: Percentage of organisms obtained from urine culture

to be more in outpatients [10]. In our study, out of 100 patients, urine culture was done only for 30 patients. In that, 18 of them (60\%) showed the isolation of E. coli followed by isolation of Proteus in 6 cases $(20 \%)$ than Klebsiella in 4 cases $(13.33 \%)$, and last is Pseudomonas in 2 cases (6.66\%).

\section{CONCLUSION}

Cephalosporins, aminoglycosides, and quinolones were the most commonly prescribed antibiotics in this study. Culture sensitivity test was carried out only in a limited number of cases, and present findings together with previous ones are suggestive of the need for periodic monitoring of antibiotic sensitivity pattern of the bacterial isolates to provide effective treatment and thereby to make it more cost effective particularly in the developing countries like India. The continuous monitoring and reporting of prescribing pattern of antibiotics will surely help the physicians for effective treatment.

\section{REFERENCES}

1. Hoffmann RP. A strategy to reduce drug expenditures with a drug utilization review program. Hosp Pharm 1984;19(1):7-8, 11-2.

2. Fallik B. The academy of managed care pharmacy's concepts in managed care pharmacy: Prior authorization and the formulary exception process. J Manag Care Pharm 2005;11(4):358-61.

3. Azzarone G, Liewehr S, O'Connor K. Cystitis. Pediatr Rev 2007;28(12):474-6.

4. Jepson RG, Craig JC. Cranberries for preventing urinary tract infections. Cochrane Database Syst Rev 2008;CD001321.

5. Huang ES, Laiteerapong N, Liu JY, John PM, Moffet HH, Karter AJ. Rates of complications and mortality in older patients with diabetes mellitus: The diabetes and aging study. JAMA Intern Med 
2014;174(2):251-8

6. Jancel T, Dudas V. Management of uncomplicated urinary tract infections. West J Med 2002;176(1):51-5.

7. Mishra B, Srivastava S, Singh K, Pandey A, Agarwal J. Symptom-based diagnosis of urinary tract infection in women: Are we over-prescribing antibiotics? Int J Clin Pract 2012;66(5):493-8

8. Edlin RS, Shapiro DJ, Hershc AL, Copp HL. Antibiotic resistance patterns of outpatient pediatric urinary tract infections. J Urol 2013;190(1):222-7.
9. Pandiamunian J, Somasundaram G. A study on prescribing pattern of anti-microbial agents in the medical intensive care unit of a tertiary care teaching hospital in Puducherry union territory, South India. Int J Pharm Pharm Sci 2014;6(3):235-8.

10. Gopal DV, Krishna TR, Kumar AS, Meda VS, Reddy KR. Prescribing pattern of antibiotics in the general medicine and paediatrics departments of a tertiary care teaching hospital. Int J Pharm Pharm Sci 2014;6(2):221-4 